\title{
Impact of Malnutrition on Treatment Outcome of Paediatric Acute Myeloid Leukemia
}

\author{
Tariq Ghafoor ${ }^{1,2}$, Shakeel Ahmed ${ }^{2}$, Sumaira Khalil ${ }^{2}$ and Tanzeela Farah ${ }^{2}$ \\ ${ }^{1}$ Department of Paediatric Oncology, Armed Forces Bone Marrow Transplant Centre, CMH Medical Complex, Rawalpindi, Pakistan \\ ${ }^{2}$ Department of Paediatric Oncology, Combined Military Hospital, Rawalpindi, Pakistan
}

\begin{abstract}
Objective: To analyse the distribution of malnutrition in paediatric acute myeloid leukaemia (AML) and its effect on treatment outcome and survival in Pakistani children.

Study Design: Descriptive, analytical study.

Place and Duration of Study: Department of Paediatric Oncology, Combined Military Hospital (CMH), Rawalpindi, Pakistan from January 2012 to January 2019.

Methodology: Data of all newly registered cases of paediatric AML was analysed to see the association of nutritional status according to Z-score classification and treatment outcome. The outcome was divided into disease-free and overall survival.

Results: A total of 206 cases, including 130 (63.1\%) boys and $76(36.9 \%)$ girls were evaluated. The mean age at diagnosis was $5.96 \pm 3.58$ years. Pallor was the most common clinical feature in 172 (83.5\%) followed by fever in $158(76.7 \%)$ cases. AML M-2 was the most frequent French-American-British (FAB) subtype in $94(45.6 \%)$ cases. Majority of the patients $(n=128,62.1 \%)$ were well nourished; and $46(22.3 \%)$ and 32 (15.5\%) were moderately and severely malnourished, respectively. Disease-free survival (DFS) was $43.0 \%, 32.6 \%$ and $21.9 \%$ in well nourished, moderately malnourished and severely malnourished patients, respectively ( $p=0.022$ ). Overall survival (OS) was $43.8 \%, 39.1 \%$ and $21.9 \%$ in well nourished, moderately malnourished and severely malnourished patients, respectively $(p=0.012)$.

Conclusion: Malnutrition adversely affects the treatment outcome in paediatric AML. It is significantly associated with increased treatment-related mortality, mainly due to infection and decreased disease-free and overall survival.
\end{abstract}

Key Words: Paediatric acute myeloid leukaemia, Mortality, Nutritional status, Weight-for-age, Z score, Pakistan.

How to cite this article: Ghafoor T, Ahmed S, Khalil S, Farah T. Impact of Malnutrition on Treatment Outcome of Paediatric Acute Myeloid Leukemia. J Coll Physicians Surg Pak 2020; 30(10):1021-1025.

\section{INTRODUCTION}

Paediatric acute myeloid leukaemia (AML) accounts for $15-25 \%$ of childhood leukaemia. The five years survival rates of paediatric AML have improved to $70 \%$. in high-income countries (HICs). This is attributed to recent advances in chemotherapy, risk-based intensive treatment and better supportive and intensive care. ${ }^{1-3}$ However, the majority of the patients live in low-and-middle-income countries (LMICS), where access to treatment is restricted. As compared to $80 \%$ survival in HICs, survival in LMICs is only $25 \% .{ }^{4}$ This poor survival rate is because of various factors, like diagnosis delay, treatment abandonment, malnutrition, inadequate supportive care and higher treatment-related mortality (TRM) ${ }^{5-7}$

Correspondence to: Dr. Tariq Ghafoor, Department of Paediatric Oncology, Armed Forces Bone Marrow Transplant Centre, $\mathrm{CMH}$ Medical Complex, Rawalpindi, Pakistan

E-mail: drtariqghafoor@gmail.com

Received: May 06, 2019; Revised: August 30, 2020;

Accepted: October 12, 2020

DOI: https://doi.org/10.29271/jcpsp.2020.10.1021
Malnutrition is often associated with increased morbidity and / or mortality in childhood cancer patients. ${ }^{8,9}$ Growth of a child is an internationally recognised parameter used as an indicator of nutritional status. ${ }^{10}$ Weight-for-age is widely used to define the growth of children and WHO recommends using Z-score to describe weight-for-age. ${ }^{11}$ This study was conducted to determine the frequency of malnutrition among paediatric AML patients and the association of malnutrition with treatment-related complications and the final outcome.

\section{METHODOLOGY}

This descriptive, analytical study was carried out at Department of Paediatric Oncology, Combined Military Hospital (CMH), Rawalpindi, Pakistan from January 2012, to January 2019. The study included all newly diagnosed cases of AML younger than 18 years, registered since January 2012 , who finished theirtreatment till $31^{\text {st }}$ January 2019. Patients having acute promyelocytic leukaemia (APL), prior chemotherapy or leaving during treatment were excluded. Study data included: age, gender, blood counts at presentation, weight-for-age, FAB classification, genetic abnormalities, chemotherapy protocol, treatment outcome, last follow-up, and cause of mortality. Detailed 
medical history and clinical examinations were performed at the first admission. Diagnosis of AML was made on the basis of blastmorphology in bonemarrowaspiration and immunophenotyping by flow cytometry. The diagnostic workup incorporated complete blood picture with differential blood cell counts, hepatic and renal function tests, uric acid, and assessment of cardiac status with echocardiography.

At the time of admission, the weight of every patient was recorded and plotted on the standard WHO Z-score charts for the weight for age and sex. The cases were classified as adequately nourished, moderately malnourished and severely malnourished, if the weight for age Z-score was $<-2$, between ${ }^{3}$ -2 to $<-3$ and $^{3}-3$, respectively.

Two courses of anthracycline-based chemotherapy either ADE (Cytarabine $100 \mathrm{mg} / \mathrm{m}^{2}$ 12-hourly on days 1-10, Daunorubicin $50 \mathrm{mg} / \mathrm{m}^{2}$ daily on days $1,3 \& 5$, and Etoposide $100 \mathrm{mg} / \mathrm{m}^{2}$ daily on days 1-5) or $\mathrm{AD}$ (Cytarabine100 $\mathrm{mg} / \mathrm{m}^{2} 12$-hourly on days 1-10 and Daunorubicin $50 \mathrm{mg} / \mathrm{m}^{2}$ daily on days $1,3 \& 5$ ) were used as induction chemotherapy course 1 . Second induction chemotherapy was same as $1^{\text {st }}$ chemotherapy course, but Cytarabine was reduced to 8 days (total 16 doses).

Two courses of high dose Cytarabine based chemotherapy (HiDAC; Cytarabine $3000 \mathrm{mg} / \mathrm{m}^{2}$ twice daily on day 1, 3 and 5) were used as consolidation therapy. Patients showing a partial response after induction chemotherapy received FLA-Ida (Fludarabine $30 \mathrm{mg} / \mathrm{m}^{2}$ daily on days 1-5, Idarubicin $10 \mathrm{mg} / \mathrm{m}^{2}$ on days $4,5 \& 6$ and Cytarabine $2000 \mathrm{mg} / \mathrm{m}^{2}$ daily on days $1-5$ ) as consolidation therapy.

All cases were admitted for the treatment. Hyper-hydration and allopurinol were used to prevent tumour lysis. Serum electrolytes, intake and output, were meticulously monitored. Clinically stable patients were discharged and followed up at least twice weekly in the outpatient clinic. They were hospitalised immediately in case of febrile neutropenia or any other complication. An absolute neutrophil count (ANC) of $<1000$ was considered as neutropenia. A single oral temperature of $>38^{\circ} \mathrm{C}$ or two readings $>37.5^{\circ} \mathrm{C}$ at least 2 hours apart were used to define fever. Broad-spectrum antibiotics were used intravenously, to treat neutropenic fever. Combination of amikacin and piperacillin-tazobactam was used as first line of antibiotics. Teicoplanin was used to treat suspected central venous line infection. Piperacillin-tazobactam was swapped with meropenem, if fever continued after 48 hours. Anti-fungal amphotericin B was added empirically, if fever continued beyond 96 hours. Red blood cell concentrate (RCC) and platelets were transfused to keep haemoglobin ${ }^{3} 8.0 \mathrm{~g} / \mathrm{dl}$ and platelets ${ }^{3} 10 \times 10^{9} / \mathrm{L}$.

Quantitative variables were expressed in average \pm standard deviation and qualitative variables in numbers and percentages. Disease-free survival (DFS) was defined as the time from the achievement of CR until relapse. Overall survival (OS) was defined as the time from the date of diagnosis till last follow-up or death from any cause. The Kaplan-Meier method was used to estimate DFS and OS; and survival differences were calculated by using Log-rank tests. Chi-squared tests were applied to document the difference in outcome among groups for each covariate. The results are shown in Table II. All statistical analyses were performed using the SPSS version 23.0, and the statistically significant $p$ value was less than 0.05 .

Table I: Patient characteristics.

\begin{tabular}{|c|c|c|}
\hline & Number (n) & Percentage (\%) \\
\hline Total number & 206 & 100 \\
\hline Age & \multicolumn{2}{|c|}{ Mean $5.96 \pm 3.57$ years (range; 9 months to 15 years) } \\
\hline Less than 5 years & 89 & 43.2 \\
\hline$>5-10$ years & 79 & 38.3 \\
\hline$>10-15$ years & 38 & 18.4 \\
\hline \multicolumn{3}{|l|}{ Gender } \\
\hline Male & 130 & 63.1 \\
\hline Female & 76 & 36.9 \\
\hline Duration of symptoms & \multicolumn{2}{|c|}{ Mean $52.64 \pm 57.69$ days (range; $1-425$ days) } \\
\hline \multicolumn{3}{|l|}{ Presentation } \\
\hline Pallor & 172 & 83.5 \\
\hline Fever & 158 & 76.7 \\
\hline Visceromegaly & 153 & 74.3 \\
\hline Bruising \& Bleeding & 105 & 51.0 \\
\hline Bone Pains & 35 & 17.0 \\
\hline Proptosis & 31 & 15.0 \\
\hline CNS Positive & 7 & 3.4 \\
\hline Granulocytic Sarcoma & 2 & 1.0 \\
\hline WBC count $\left(\times 10^{9} / \mathrm{L}\right)$ & \multicolumn{2}{|c|}{ Mean $55.67 \pm 68.45$ (Range; 1.1-408) } \\
\hline$\left(<50 \times 10^{9} / \mathrm{L}\right)$ & 129 & 62.6 \\
\hline$\left(>50 \times 10^{9} / \mathrm{L}\right)$ & 77 & 37.4 \\
\hline Haemoglobin (g/dl) & \multicolumn{2}{|c|}{ Mean 7.59 \pm 2.53 (Range; $2.9-15.9$ ) } \\
\hline Platelets $\left(\times 10^{9} / \mathrm{L}\right)$ & \multicolumn{2}{|c|}{ Mean 55.51 \pm 78.40 (Range; 2-684) } \\
\hline \multicolumn{3}{|l|}{ FAB Classification } \\
\hline AML-MO & 17 & 8.3 \\
\hline AML-M1 & 22 & 10.7 \\
\hline AML-M2 & 94 & 45.6 \\
\hline AML-M4 & 26 & 12.6 \\
\hline AML-M5 & 11 & 5.3 \\
\hline AML-M6 & 4 & 1.9 \\
\hline AML-M7 & 5 & 2.4 \\
\hline AML-DS & 4 & 1.9 \\
\hline AML-NOS & 23 & 11.2 \\
\hline
\end{tabular}

\section{RESULTS}

A total of 255 new cases of AML were registered during the study period. Forty-nine cases (including 39 cases of APL), three cases that refused treatment and seven cases, who were on active treatment were excluded from the study.

Data of 206 cases, including 130 (63.1\%) males and 76 (36.9\%) females were analysed. The mean age at diagnosis was $5.96 \pm$ 3.58 years. The most common presenting feature was pallor in $172(83.5 \%)$, followed by fever in $158(76.7 \%)$ cases. Physical examination revealed pallor in 172 (83.5\%) patients followed by visceromegaly in $153(74.3 \%)$ patients. The mean white blood cells count was $55.67 \pm 68.46 \times 10^{9} /$ l. Initial WBC of $>50 \times 10^{9} / \mathrm{L}$ was seen in 77 (37.4\%) patients. The mean haemoglobin was $7.60 \pm$ $2.54 \mathrm{~g} / \mathrm{dl}$, and the mean platelets count was $55.52 \pm 78.41 \times 10^{9} / \mathrm{l}$. Only seven (3.4\%) patients had CNS (central nervous system) disease. AML M-2 was the commonest FAB subtype in 94 (45.6\%) cases, followed by AML M-4 in 26 (12.6\%) cases (Table I).Six (2.9\%) patients died before the start of chemotherapy. Out of the remaining 200 cases, 114 (57.0\%) patients had ADE chemotherapy and $86(43.0 \%)$ had AD chemotherapy. The result of $1^{\text {st }}$ chemotherapy was TRM $43(21.5 \%)$ cases, complete morphological remission (CR) 117 (58.5\%) cases, partial response (PR) 25 $(12.5 \%)$ and resistant/refractory disease (RD) 15 (7.5\%) cases. 
Table II: Survival according to nutritional status in AML patients.

\begin{tabular}{|c|c|c|c|c|c|}
\hline Variables & Well nourished & Moderately malnourished & Severely malnourished & Total & p-value \\
\hline TRM & $32(25.0 \%)$ & $15(32.6 \%)$ & $18(56.3 \%)$ & $65(31.6 \%)$ & 0.003 \\
\hline RD & $41(32.0 \%)$ & $16(34.8 \%)$ & $7(21.9 \%)$ & $64(31.1 \%)$ & 0.44 \\
\hline DFS & $55(43.0 \%)$ & $15(32.6 \%)$ & $7(21.9 \%)$ & $77(37.4 \%)$ & 0.022 \\
\hline OS & $56(43.0 \%)$ & $18(39.1 \%)$ & $7(21.9 \%)$ & $88(39.3 \%)$ & 0.012 \\
\hline Total & 128 & 46 & 32 & 206 & \\
\hline
\end{tabular}
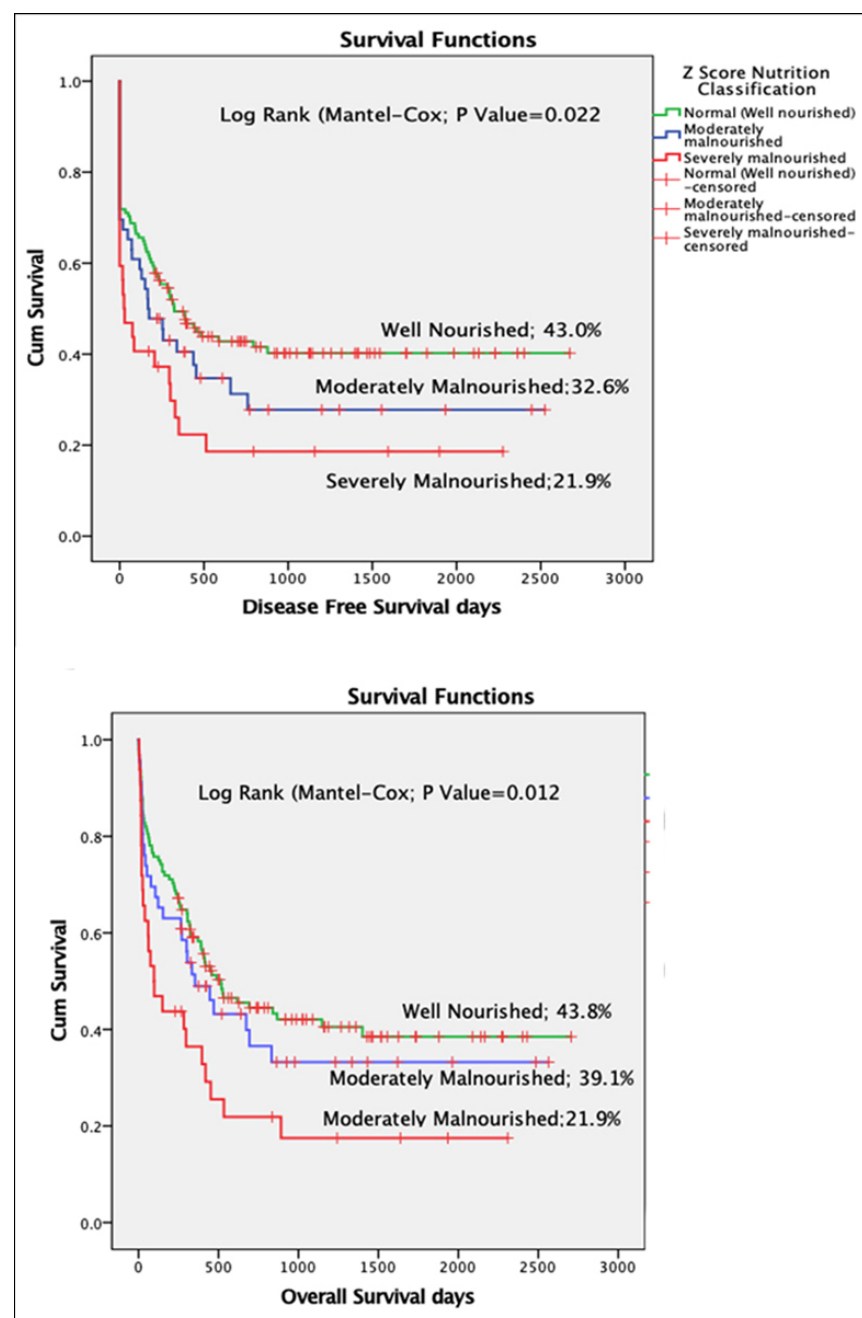

Figure 1: Disease-free and overall survival in AML, according to the nutritional status of the patient.

One hundred and fifty-four patients received the second course of chemotherapy. After two courses of induction chemotherapy; TRM was 54 (27.0\%), CR 130 (65.0\%), PR 11 (5.5\%) and RD 4 (2.0\%).

In the present cohort overall, non-relapse mortality (NRM) was $65 / 206(31.6 \%)$, including $6 / 206(2.9 \%)$ deaths before the start of chemotherapy, 43/200 (21.5\%), 11/154 (7.1\%) and 5/139 (3.6\%) during first, second, and third chemotherapy courses, respectively. No patient died during the fourth course of chemotherapy. Neutropenic fever and bleeding were the two most common causes of NRM. Out of nine bleeding related deaths, five had GI bleeding, three had intracranial bleeding and one case had pulmonary haemorrhage. A total of 130 cases completed full treatment. Sixty-four cases had the refractory or relapsed disease; and mortality in the relapsed group was 60 (93.8\%).

According to Z-score classification, the majority of the patients, $128(62.1 \%)$ were well nourished, $46(22.3 \%)$ and $32(15.5 \%)$ were moderately and severely malnourished, respectively Out of 128 well-nourished patients, 32 (25\%) had TRM, 41 (32.0\%) had relapse-related (RD) mortality and DFS was 55 (43.0\%). TRM was statistically significantly high in severely malnourished children $(p=0.03)$. Among the 46 moderately malnourished patients, $28 / 46(60.9 \%)$ patients died including 15 (32.6\%) deaths during treatment, while 13 patients $(28.3 \%)$ died due to relapse/resistant disease. Among 32 severely malnourished, $18(56.3 \%)$ patients had TRM while 7 (21.9\%) patients died due to relapse/resistant disease and DFS was 7 (21.9\%), Table II.

One hundred and twenty-five (60.7\%) patients died during the study period and major causes of mortality were neutropenic sepsis (36), respiratory failure (15), bleeding (9), hyperleukocytosis (5) and resistant/relapsed disease (60).

DFS and OS differed significantly among the three groups. DFS was $43.0 \%, 32.6 \%$ and $21.9 \%$ in well nourished, moderately malnourished and severely malnourished patients, respectively $(p=0.022)$. OS was $43.8 \%, 39.1 \%$ and $21.9 \%$ in well nourished, moderately malnourished and severely malnourished patients, respectively $(p=0.012$, Figure 1$)$.

\section{DISCUSSION}

Five-year survival rate of paediatric AML now approach $70 \%$ in high-income countries (HICS). ${ }^{1,12}$ However, the majority of childhood cancer patients live in resource-limited countries; and malnutrition is rampant in these societies. ${ }^{13}$ Malnutrition results in increased morbidity and mortality in patients of paediatric leukaemia. ${ }^{14,15}$ Cancer and its treatment worsen the nutritional status of already malnourished children due to anorexia, inflammation and increased metabolic rate. ${ }^{16}$ In the present study, over one-third of patients were malnourished. A similar high rate of malnutrition at the time of diagnosis, have been reported by various other studies in children from other LMICs. ${ }^{9,13,17}$

In the present study, like other LMICs, TRM was quite high, especially in severely undernourished children. TRM was $25 \%$ in well-nourished children and increased to $56.3 \%$ in 
severely malnourished patients. Infection alone or with associated bleeding and respiratory failure were the main causes of TRM, which and mainly occurred during the induction period. This association of infection-related high mortality with malnutrition supports the findings reported by various previous studies. ${ }^{13,15}$ Higher TRM in underweight patients has also been reported from developed counties. ${ }^{18}$ This high mortality can be explained on the basis of the fact that malnutrition worsens in cancer patients because of an increased metabolic rate due to disease and neutropenic fever. ${ }^{16}$ Moreover, anorexia secondary to chemotherapy-induced nausea and vomiting, and mucositis results in decreased oral intake. Decreased availability of the food of choice in the hospital also results in decreased oral intake. Malnutrition in Malawian patients with Wilms tumour and other LMICs has been managed with nutritional interventions. $^{19}$

Parenteral nutrition for paediatric patients was not available at the study centre. Though commercially available oral nutritional supplements were advised to children, but many of them were not very eager to take them. Malnutrition results in decreased complement, cytokines, and immunoglobulins, leading to impaired immune system functions. Correction of nutrition deficiencies, infusion of supplemental immunoglobulin and prophylactic antibiotics are recommended for undernourished children undergoing chemotherapy for AML. ${ }^{18}$ NG feeds can be helpful during periods of chemotherapy-related nausea and vomiting.

OS and DFS were statistically significantly lower in malnourished patients. This was mainly due to increased TRM in malnourished children. However, relapse-related mortality was found to be inversely proportionate to the degree of malnutrition. This may raise a hypothesis for future researches that malnutrition decreases the chances of relapse-related deaths in paediatric AML patients.

\section{CONCLUSION}

Malnutrition adversely affects the treatment outcome in paediatric AML. It is associated with increased treatment-related mortality, mainly due to infection and decreased disease-free and overall survival.

\section{ETHICAL APPROVAL:}

The research work for this study was commenced after the approval from the Hospital Ethical Committee.

\section{PATIENTS' CONSENT:}

Informed consents were obtained from parents of the patients to publish the data for this study.

\section{CONFLICT OF INTEREST:}

The authors declare no conflict of interest or financial interest in any product or service mentioned in the manuscript, including grants, equipment, medications, employment, gifts, and honoraria.

\section{AUTHORS' CONTRIBUTIONS:}

TG: Conception and design, interpretation, critical revision and final approval.

SA: Data acquisition and analysis, interpretation, drafting, and final approval.

SK, TF: Interpretation, critical revision, and final approval.

\section{REFERENCES}

1. Taga T, Tomizawa D, Takahashi H, Adachi S. Acute myeloid leukemia in children: Current status and future directions. Pediatr Int 2016; 58(2):71-80. doi: 10.1111/ped.12865.

2. Wennström L, Edslev PW, Abrahamsson J, Nørgaard JM, Fløisand $Y$, Forestier $E$, et al. Acute myeloid leukemia in adolescents and young adults treated in paediatric and adult departments in the nordic countries. Pediatr Blood Cancer 2016; 63(1):83-92. doi: 10.1002/pbc.25713.

3. Creutzig U, Zimmermann M, Reinhardt D, Dworzak M, Stary J, Lehrnbecher T. Early deaths and treatment-related mortality in children undergoing therapy for acute myeloid leukemia: Analysis of the multicenter clinical trials AML-BFM 93 and AML-BFM 98. J Clin Oncol 2004; 22(21): 4384-93. doi: 10.1200/JCO.2004.01.191.

4. Kellie SJ, Howard SC. Global child health priorities: What role for paediatric oncologists? Eur J Cancer 2008; 44(16):2388-96. doi: 10.1016/j.ejca.2008.07.022.

5. Gupta S, Bonilla M, Valverde P, Fu L, Howard SC, Ribeiro RC, et al. Treatment-related mortality in children with acute myeloid leukaemia in Central America: incidence, timing and predictors. Eur J Cancer 2012; 48(9):1363-9. doi: 10.1016/j.ejca.2011.10.009.

6. Fadoo Z, Mushtaq N, Alvi S, Ali M. Acute myeloid leukaemia in children: Experience at a tertiary care facility of Pakistan. J Pak Med Assoc 2012; 62(2):125-8.

7. Asim M, Zaidi A, Ghafoor T, Qureshi Y. Death analysis of childhood acute lymphoblastic leukaemia; experience at Shaukat Khanum Memorial Cancer Hospital and Research Centre, Pakistan. J Pak Med Assoc 2011; 61(7):666-70.

8. Hingorani $P$, Seidel K, Krailo M, Mascarenhas L, Meyers $P$, Marina N, et al. Body mass index (BMI) at diagnosis is associated with surgical wound complications in patients with localized osteosarcoma: A report from the children's oncology group. Pediatr Blood Cancer 2011; 57(6):939-42. doi: 10.1002/pbc.23129.

9. Sala A, Rossi E, Antillon F, Molina AL, de Maselli T, Bonilla M, et al. Nutritional status at diagnosis is related to clinical outcomes in children and adolescents with cancer: A perspective from Central America. Eur J Cancer 2012; 48(2):243-52. doi: 10.1016/j.ejca.2011.06.006.

10. Srivastava A, Mahmood SE, Srivastava PM, Shrotriya VP, Kumar B. Nutritional status of school-age children - A scenario of urban slums in India. Arch Public Health 2012; 70(1):8. doi: 10.1186/0778-7367-70-8.

11. http://www.who.int/nutgrowthdb/about/introduction/en/inde x5.html.

12. Creutzig U, Van den Heuvel-Eibrink MM, Gibson B, Dworzak MN, Adachi S, de Bont E, et al. Diagnosis and management of acute myeloid leukemia in children and adolescents: Recommendations from an international expert panel. Blood 
2012; 120(16):3187-205. doi: 10.1182/blood-2012-03362608 .

13. Pribnow AK, Ortiz R, Báez LF, Mendieta L, Luna-Fineman S. Effects of malnutrition on treatment-related morbidity and survival of children with cancer in Nicaragua. Pediatr Blood Cancer 2017; 64(11):e26590. doi: 10.1002/pbc.26590.

14. Khan AR, Sheikh MH, Intekhab K. Pre-existing malnutrition and treatment outcome in children with acute lymphoblastic leukaemia. J Pak Med Assoc 2006; 56(4): 171-3.

15. Barr RD, Gomez-Almaguer D, Jaime-Perez JC, Ruiz-Argüelles GJ. Importance of nutrition in the treatment of leukemia in children and adolescents. Arch Med Res 2016; 47(8): 585-92. doi: 10.1016/j.arcmed.2016.11.013.

16. Brinksma A, Huizinga G, Sulkers E, Kamps W, Roodbol P, Tissing W. Malnutrition in childhood cancer patients: A review on its prevalence and possible causes. Crit Rev Oncol Hematol 2012; 83(2):249-75. doi: 10.1016/ j.critrevonc.2011.12.003.

17. Bauer J, Jürgens $\mathrm{H}$, Frühwald $\mathrm{MC}$. Important aspects of nutrition in children with cancer. Adv Nutr 2011; 2(2):67-77. doi: 10.3945/an.110.000141.

18. Inaba H, Surprise HC, Pounds S, Cao X, Howard SC, Ringwald-Smith $\mathrm{K}$, et al. Effect of body mass index on the outcome of children with acute myeloid leukemia. Cancer 2012; 118(23):5989-96. doi: 10.1002/cncr.27640.

19. Israëls $T$, Borgstein E, Jamali $M$, de Kraker J, Caron HN, Molyneux EM. Acute malnutrition is common in Malawian patients with a wilms tumour: A role for peanut butter. Pediatr Blood Cancer 2009; 53(7):1221-6. doi: 10.1002/ pbc.22158. 